\title{
FIXED POINTS OF MAPPINGS SATISFYING SEMICONTRACTIVITY CONDITIONS
}

\author{
JÜRGEN SCHU \\ (Received 6 June 1990; revised 27 November 1990) \\ Communicated by P. G. Dodds
}

\begin{abstract}
Let $A$ be a subset of a Banach space $E$. A mapping $T: A \rightarrow A$ is called asymptotically semicontractive if there exists a mapping $S: A \times A \rightarrow A$ and a sequence $\left(k_{n}\right)$ in $[1, \infty)$ such that $T x=S(x, x)$ for all $x \in A$ while for each fixed $x \in A, S(., x)$ is asymptotically nonexpansive with sequence $\left(k_{n}\right)$ and $S(x,$.$) is strongly compact. Among other things, it is proved that each asymptotically semi-$ contractive self-mapping $T$ of a closed bounded and convex subset $A$ of a uniformly convex Banach space $E$ which satisfies Opial's condition has a fixed point in $A$, provided $s$ has a certain asymptotic regularity property.
\end{abstract}

1991 Mathematics subject classification (Amer. Math. Soc.): 47 H 10.

Keywords and phrases: Fixed point, semicontractive mapping, asymptotically nonexpansive mapping, asymptotically semicontractive mapping, uniformly convex Banach space, uniformly smooth Banach space, weakly sequentially continuous duality mapping, Opial's condition.

\section{Introduction}

A self-mapping $T$ of a nonempty subset $A$ of a Banach space $E$ is called semicontractive (weakly semicontractive) if there exists a mapping $S: E \times$ $E \rightarrow A$ such that $T x=S(x, x)$ for all $x \in A$ while for each fixed $x \in$ $E, S(., x)$ is nonexpansive and $S(x,$.$) is strongly compact (compact). It$ is shown in [3] that each semicontractive self-mapping $T$ of a nonempty closed bounded and convex subset $A$ of a reflexive Banach space $E$ which possesses a weakly sequentially continuous duality mapping has a fixed point

(C) 1992 Australian Mathematical Society 0263-6115/92 \$A2.00+0.00 
in $A$. This result in particular applies to $l_{p}, p \in(1, \infty)$, but not to $L_{p}$, $p \in(1, \infty) \backslash\{2\}$.

In Section 1, we show among other things that this result remains valid if we replace the assumption " $S(., x)$ is nonexpansive for each $x \in E$ " with the weaker one " $S(., x)$ is asymptotically nonexpansive with sequence $\left(k_{n}\right)$ for each $x \in A$ and some fixed sequence $\left(k_{n}\right) \in[1, \infty)^{\mathrm{N}}$, provided $S$ satisfies a certain asymptotic regularity condition and $E$ is additionally supposed to be uniformly convex (see Theorem 1.12). The class of asymptotically nonexpansive mappings was introduced by K. Goebel and W.A. Kirk [5] in 1972 , and since then has been studied in various papers (see, for example, $[1,7,9,11,14,15])$.

In Section 2, we consider a uniformly smooth Banach space and prove a fixed point theorem for weakly semicontractive mappings satisfying a strengthened compactness condition. This result, which applies both to the $l_{p}$ and the $L_{p}$ spaces $(p \in(1, \infty))$, was motivated by the main result of Browder [3] concerning weakly semicontractive mappings. Browder's result, however, does not apply to $L_{p}(p \in(1, \infty) \backslash\{2\})$ because the underlying space is supposed to possess a weakly sequentially continuous duality mapping there.

\section{Preliminaries}

A normed space $(E,\|\cdot\|)$ is called uniformly convex if for each $\epsilon>0$ there exists a $\delta>0$ such that if $x, y \in E$ with $\|x\|,\|y\| \leq 1$ and $\|x-y\| \geq \epsilon$ it follows that $\|x+y\| \leq 2(1-\delta)$. We call $(E,\|\cdot\|)$ (uniformly) smooth if the norm of $E$ is (uniformly) Gâteaux-differentiable on the boundary of the unit ball in $E$. Furthermore, $(E,\|\cdot\|)$ is said to satisfy Opial's condition if for each sequence $\left(x_{n}\right) \in E^{\mathbb{N}}$ which converges weakly to some $x \in E$, it follows that $\liminf \left\|x_{n}-x\right\|<\liminf \left\|x_{n}-y\right\|$ for all $y \in E \backslash\{x\}$.

For a given gauge function $\mu$, this means for a mapping $\mu: \mathbb{R}^{+} \rightarrow \mathbb{R}^{+}$ which is continuous and strictly increasing with $\mu(0)=0$ and $\lim _{x \rightarrow \infty} \mu(x)$ $=\infty$, the related set-valued duality mapping $J_{E}^{\mu}: E \rightarrow 2^{E^{*}}$ is given by

$$
J_{E}^{\mu}(x)=\left\{u \in E^{*} \mid u(x)=\|u\|\|x\| \text { and }\|u\|=\mu(\|x\|)\right\}
$$

for all $x \in E$. A mapping $J: E \rightarrow E^{*}$ is called a duality mapping with respect to $\mu$ if $J(x) \in J_{E}^{\mu}(x)$ for all $x \in E$. Such a mapping $J$ is said to be weakly sequentially continuous if for all $\left(x_{n}\right) \in E^{\mathbb{N}}$ and all $x \in E$ it follows from $\left(x_{n}\right) \rightarrow x$ that $\left(J\left(x_{n}\right)\right) \doteq J(x)$ (as usual $\rightarrow$ and \pm stand for weak and weak ${ }^{*}$ convergence, respectively, while strong convergence of a sequence $\left(z_{n}\right)$ to a point $z$ is indicated by $\lim \left(z_{n}\right)=z$ ). It is well-known 
that $J_{E}^{\mu}$ is single-valued if and only if $(E,\|\cdot\|)$ is smooth (see, for example, [4, page 22]). In this case we regard $J_{E}^{\mu}$ as a mapping from $E$ to $E^{*}$.

In all our proofs we assume, without loss of generality, that $\mu=$ id (the identity mapping). For abbreviation we set $J_{E}=J_{E}^{\text {id }}$. Furthermore, in the sequel $E$ is always assumed to be a linear space over the real field.

\section{Fixed points of asymptotically semicontractive and weakly asymptotically semicontractive mappings}

Definition 1.1. Let $(E,\|\cdot\|)$ be a normed space, $\varnothing \neq A \subset E$ and $T: A \rightarrow A$.

(a) $T$ is called Lipschitzian with constant $L \geq 0$ if $\|T x-T y\| \leq$ $L\|x-y\|$ for all $x, y \in A$.

(b) $T$ is called nonexpansive (a Banach-contraction) if $T$ is Lipschitzian with constant $L=1 \quad(L<1)$.

(c) $T$ is called compact if $T$ is continuous and maps bounded sets onto relatively compact ones.

(d) $T$ is called strongly compact (or completely continuous) if $T$ is continuous from the weak topology of $E$ to the strong topology of $E$.

(e) $T$ is called asymptotically nonexpansive with sequence $\left(k_{n}\right) \in$ $[1, \infty)^{\mathbf{N}}$ if $\lim \left(k_{n}\right)=1$ and $\left\|T^{n}(x)-T^{n}(y)\right\| \leq k_{n}\|x-y\|$ for all $n \in \mathbb{N}$ and all $x, y \in A$.

Our next definition follows the corresponding one of [3]. Note, however, that, contrary to F. E. Browder, we do not demand that $S$ is defined on the whole of $E \times E$.

Definition 1.2. Let $(E,\|\cdot\|)$ be a normed space, $\varnothing \neq A \subset E$ and $T: A \rightarrow A$.

(a) $T$ is called asymptotically semicontractive if there exists a mapping $S: A \times A \rightarrow A$ and a sequence $\left(k_{n}\right) \in[1, \infty)^{\mathbb{N}}$ such that $T x=S(x, x)$ for all $x \in A$ while for each fixed $x \in A, S(., x)$ is asymptotically nonexpansive with sequence $\left(k_{n}\right)$ and $S(x,$.$) is strongly compact.$

(b) $T$ is called weakly asymptotically semicontractive if there exists a mapping $S: A \times A \rightarrow A$ and a sequence $\left(k_{n}\right) \in[1, \infty)^{\mathbb{N}}$ such that $T x=S(x, x)$ for all $x \in A$ while for fixed $x \in A, S(., x)$ is asymptotically nonexpansive with sequence $\left(k_{n}\right)$ and, for fixed $x \in A$ and fixed $n \in \mathbb{N}$, the mapping $y \rightarrow S(., y)^{n}(x)$ is compact on $A$.

REMARK. Clearly every semicontractive mapping is asymptotically semicontractive too. The example below in particular shows that the converse is 
not true. Following Goebel and Kirk [5] we define $f: l_{2} \rightarrow l_{2}$ as follows:

$$
f(x)=\left(0, x_{1}^{2}, a_{2} x_{2}, a_{3} x_{3}, \ldots\right)
$$

where $\left(a_{n}\right) \in(0,1)^{\mathrm{N}}$ is such that $\prod_{n=2}^{\infty} a_{n}=\frac{1}{2}$. Furthermore we define $g, \widehat{g}: l_{2} \rightarrow l_{2}$ according to

$$
g(x)=\left(x_{1}, 0,0,0, \ldots\right) \quad \text { and } \quad \widehat{g}(x)=(\|x\|, 0,0,0, \ldots) .
$$

Finally let $T, \widehat{T}: l_{2} \rightarrow l_{2}$ be defined by

$$
T=f+g \quad \text { and } \quad \widehat{T}=f+\widehat{g} .
$$

Then

(i) $T$ is asymptotically semicontractive.

(ii) $\widehat{T}$ is weakly asymptotically semicontractive.

Additionally it can be shown that $T$ is neither semicontractive nor asymptotically nonexpansive nor strongly compact. Furthermore $\widehat{T}$ is neither asymptotically semicontractive nor weakly semicontractive nor asymptotically nonexpansive nor compact.

In order to prove (i) and (ii) we define mappings $S, \widehat{S}: E \times E \rightarrow E$ according to $S(x, y)=f(x)+g(y)$ and $\widehat{S}(x, y)=f(x)+\widehat{g}(y)$ and denote the standard basis of $l_{2}$ by $\left\{e_{n} \mid n \in \mathbb{N}\right\}$. Then $T(x)=S(x, x)$ and $\widehat{T}(x)=$ $\widehat{S}(x, x)$ for all $x \in E$, and

$$
f^{n}(z)=z_{1}^{2}\left(\prod_{j=2}^{n} a_{j}\right) e_{n+1}+\sum_{i=n+1}^{\infty} z_{i+1-n}\left(\prod_{j=i+1-n}^{i} a_{j}\right) e_{i+1}
$$

for all $n \in \mathbb{N}$ and all $z \in E$. Now, define

$$
b_{n}(\alpha)=\alpha e_{1}+\alpha^{2} e_{2}+\alpha^{2} \sum_{i=2}^{n-1}\left(\prod_{j=2}^{i} a_{j}\right) e_{i+1}
$$

for all $n \in \mathbb{N}$ and all $\alpha \in \mathbb{R}$. Then, for each fixed $x \in E$, all $n \in \mathbb{N}$ and all $z \in E$,

$$
S(\cdot, x)^{n}(z)=b_{n}\left(x_{1}\right)+f^{n}(z)
$$

and thus, for all $n \in \mathbb{N}$ and all $z, w \in E$,

$$
S(\cdot, x)^{n}(z)-S(\cdot, x)^{n}(w)=f^{n}(z)-f^{n}(w) .
$$

From [5] we know that $f$ is asymptotically nonexpansive with sequence $\left(k_{n}\right) \in[1, \infty)^{\mathbf{N}}$. Thus it follows from the equation above that for each fixed $x \in E$ the mapping $S(\cdot, x)$ is asymptotically nonexpansive with the same sequence $\left(k_{n}\right)$. Furthermore, $g$ is clearly strongly compact, which in turn implies that for each fixed $x \in E$ the mapping $S(x, \cdot): z \rightarrow f(x)+g(z)$ is strongly compact too. This finishes the proof of (i). 
To prove (ii) first observe that for each fixed $x \in E$ the mapping $\widehat{S}(\cdot, x)$ is asymptotically nonexpansive with sequence $\left(k_{n}\right)$. This follows by an argument completely analogous to the one used in case of $S$ (just replace $x_{1}$ with $\|x\|)$. Fix $x \in E$ and $n \in \mathbb{N}$ now, and denote the mapping $z \rightarrow \widehat{S}(\cdot, z)^{n}(x)$ by $F$. Then, for all $z \in E, F(z)=b_{n}(\|z\|)+f^{n}(x)$, from which it follows that $F$ maps bounded sets onto bounded sets. This, together with the fact that

$$
\operatorname{dim}\left(\Sigma\left\{F(z)-f^{n}(x) \mid z \in E\right\}\right)=\operatorname{dim}\left(\Sigma\left\{b_{n}(\|z\|) \mid z \in E\right\}\right) \leq n<\infty
$$

shows that $F$ maps bounded sets onto relatively compact ones. Thus $F$ is compact, which completes the proof of (ii).

Lemma 1.3. Let $(E,\|\cdot\|)$ be a Banach space, $\varnothing \neq A \subset E$ closed and $\lambda \in[0,1)$. Suppose $S: A \times A \rightarrow A$ is such that $\left\|S\left(y_{1}, x\right)-S\left(y_{2}, x\right)\right\| \leq$ $\lambda\left\|y_{1}-y_{2}\right\|$ for all $x, y_{1}, y_{2} \in A$. Then

(a) there is exactly one mapping $R: A \rightarrow A$ such that $S(R x, x)=R x$ for all $x \in A$,

(b) $\|R z-R w\| \leq\left(\frac{1}{1-\lambda}\right)\|S(R w, z)-S(R w, w)\|$ for all $z, w \in A$.

Proof. For each $x \in A$ the mapping $S(., x): A \rightarrow A$ is a Banachcontraction and thus has a unique fixed point $R x$ in $A$ by the Banach fixed point theorem. This establishes (a). To show (b), fix $z, w \in A$. Then

$$
\begin{gathered}
\|R z-R w\| \leq\|S(R z, z)-S(R w, z)\|+\|S(R w, z)-S(R w, w)\| \\
\leq \lambda\|R z-R w\|+\|S(R w, z)-S(R w, w)\| .
\end{gathered}
$$

Since $\lambda \in[0,1)$, this leads to the desired inequality.

The following lemma is an improvement of a result contained in [3, Lemma 4]. In this lemma F. E. Browder assumes that $S$ is defined on $E \times E$ and that for each fixed $x \in E, S(x,$.$) is compact on A$ and $S(., x)$ is a Banachcontraction on the whole of $E$. We emphasize that there is no need for us to assume that $S$ is defined outside of $A \times A$.

LEMMA 1.4. Let $(E,\|\cdot\|)$ be a reflexive Banach space possessing a weakly sequentially continuous duality mapping $J: E \rightarrow E^{*}$ and $\varnothing \neq A \subset E$ closed bounded and convex. Suppose $S: A \times A \rightarrow A$ is such that

(i) $\left\|S\left(y_{1}, x\right)-S\left(y_{2}, x\right)\right\| \leq \lambda\left\|y_{1}-y_{2}\right\|$ for all $x, y_{1}, y_{2} \in A$ and some fixed $\lambda \in[0,1)$,

(ii) $S(x,$.$) is compact for each x \in A$. Then there exists an $x \in A$ such that $S(x, x)=x$.

Proof. Let $R: A \rightarrow A$ be the mapping given by part (a) of Lemma 1.3. Since $S(x,$.$) is continuous for each x \in A$ by (ii), it follows from part (b) 
of Lemma 1.3 that $R$ is continuous too. Thus, it remains to show that $R(A)$ is relatively compact, because then it follows from the Schauder fixed point theorem that there exists an $x \in A$ such that $R x=x$, which in turn implies that $S(x, x)=x$, taking into account that $S(R x, x)=R x$.

Therefore, fix $\left(x_{n}\right) \in A^{\mathbf{N}}$ and set $y_{n}=R\left(x_{n}\right)$ for all $n \in \mathbb{N}$. Since $E$ is reflexive, $A$ is weakly compact and thus $\left(y_{n}\right)$ possesses some subsequence $\left(y_{\varphi_{n}}\right)$ which converges weakly to a point $y \in A$. Additionally, by (ii), there exists a subsequence $\left(x_{\varphi_{\psi_{n}}}\right)$ of $\left(x_{\varphi_{n}}\right)$ and a point $z \in E$ such that $\lim S\left(y, x_{\varphi_{\psi_{n}}}\right)=z$. For abbreviation we denote $\varphi \circ \psi$ by $\mu$. Since $J$ is weakly sequentially continuous, we have $\left(J\left(y_{\mu_{n}}-y\right)\right) \div J(0)$ and thus

$$
\lim _{n \rightarrow \infty} J\left(y_{\mu_{n}}-y\right)\left(S\left(y, x_{\mu_{n}}\right)-y\right)=J(0)(z-y)=0 .
$$

Additionally, since $y_{n}=S\left(y_{n}, x_{n}\right)$,

$$
\begin{aligned}
& \left\|y_{n}-y\right\|^{2}=J\left(y_{n}-y\right)\left(S\left(y_{n}, x_{n}\right)-y\right) \\
& \quad=J\left(y_{n}-y\right)\left(S\left(y, x_{n}\right)-y\right)+J\left(y_{n}-y\right)\left(S\left(y_{n}, x_{n}\right)-S\left(y, x_{n}\right)\right) \\
& \quad \leq J\left(y_{n}-y\right)\left(S\left(y, x_{n}\right)-y\right)+\lambda\left\|y_{n}-y\right\|^{2},
\end{aligned}
$$

for all $n \in \mathbb{N}$. Since $\lambda \in[0,1)$, this implies that, for all $n \in \mathbb{N}$,

$$
\left\|y_{n}-y\right\|^{2} \leq\left(\frac{1}{1-\lambda}\right) J\left(y_{n}-y\right)\left(S\left(y, x_{n}\right)-y\right) \text {. }
$$

It follows from (1) and (2) that $\lim \left\|y_{\mu_{n}}-y\right\|=0$. Thus $R(A)$ is relatively compact.

REMARK. It is not difficult to see that, as a consequence of Lemma 1.4, in [3, Theorem 1 and 2], as well as in all the results of [13], it is possible to restrict the domain of definition of the mappings corresponding to $S$ above to $A \times A$.

THEOREM 1.5. Let $(E,\|\cdot\|)$ be a reflexive Banach space possessing a weakly sequentially continuous duality mapping and $\varnothing \neq A \subset E$ closed bounded and convex. Suppose $T: A \rightarrow A$ is weakly asymptotically semicontractive with data $\left(S,\left(k_{n}\right)\right)$ and satisfies the following condition:

(R) for each $\epsilon>0$ there exists an $n_{0} \in \mathbb{N}$ such that for all $n \geq n_{0}$ and all $z \in A$,

$$
\left\|S(., z)^{n+1}(z)-S(., z)^{n}(z)\right\|<\epsilon .
$$

Then

(a) $\inf \{\|x-T x\| \mid x \in A\}=0$,

(b) if $(\mathrm{id}-T)(A)$ is closed, it follows that $\operatorname{Fix}(T) \neq \varnothing$. 
$($ Fix $(T)$ denotes the fixed point set of $T$.)

Proof. Without loss of generality we may assume that $0 \in A$. Define $\lambda_{n}=1-1 / n$ for all $n \in \mathbb{N}$. Then, since $A$ is convex, the mapping $S_{n}$ given by $S_{n}(x, y)=\left(\lambda_{n} / k_{n}\right) S(., y)^{n}(x)$ maps $A \times A$ into $A$ for each fixed $n \in \mathbb{N}$. Additionally, for fixed $x \in A, S_{n}(x,$.$) is compact and S_{n}(., x)$ is a Banachcontraction with Lipschitz constant $\lambda_{n}$. This follows immediately from the weak asymptotic semicontractivity of $T$. Thus, by Lemma 1.4, for each $n \in$ $\mathbb{N}$ there exists an $x_{n} \in A$ such that $x_{n}=S_{n}\left(x_{n}, x_{n}\right)=\left(\lambda_{n} / k_{n}\right) S\left(., x_{n}\right)^{n}\left(x_{n}\right)$. Hence ||$x_{n}-S\left(., x_{n}\right)^{n}\left(x_{n}\right)|| \leq\left|1-\left(k_{n} / \lambda_{n}\right)\right| \operatorname{diam}(A)$ for all $n \in \mathbb{N}$, and so

$$
\lim _{n \rightarrow \infty}\left\|x_{n}-S\left(., x_{n}\right)^{n}\left(x_{n}\right)\right\|=0 .
$$

Additionally, by (R),

$$
\lim _{n \rightarrow \infty}\left\|S\left(., x_{n}\right)^{n}\left(x_{n}\right)-S\left(., x_{n}\right)^{n-1}\left(x_{n}\right)\right\|=0 .
$$

Furthermore, for all $n \in \mathbb{N}$,

$$
\begin{aligned}
\| x_{n}- & T x_{n}\|\leq\| x_{n}-S\left(., x_{n}\right)^{n}\left(x_{n}\right)\|+\| S\left(., x_{n}\right)^{n}\left(x_{n}\right)-S\left(., x_{n}\right)\left(x_{n}\right) \| \\
\leq & \left\|x_{n}-S\left(., x_{n}\right)^{n}\left(x_{n}\right)\right\|+k_{1}\left\|S\left(., x_{n}\right)^{n-1}\left(x_{n}\right)-x_{n}\right\| \\
\leq & \left\|x_{n}-S\left(., x_{n}\right)^{n}\left(x_{n}\right)\right\| \\
& +k_{1}\left(\left\|S\left(., x_{n}\right)^{n-1}\left(x_{n}\right)-S\left(., x_{n}\right)^{n}\left(x_{n}\right)\right\|+\left\|S\left(., x_{n}\right)^{n}\left(x_{n}\right)-x_{n}\right\|\right) .
\end{aligned}
$$

This, together with (3) and (4), implies that $\lim \left\|x_{n}-T x_{n}\right\|=0$, which establishes (a). Claim (b) is a direct consequence of (a).

REMARK. The assumption $(\mathbf{R})$ is a certain uniform asymptotic regularity condition for the mapping $S$. This condition, with $f$ instead of $S(., z)$, has been used by P. Vijayaraju in [15] in order to establish the existence of a fixed point of an asymptotically nonexpansive mapping $f$ with the help of almost fixed points $z_{n}=\mu_{n} f^{n}\left(z_{n}\right)$, as well as in [14] in connection with the iterative approximation of fixed points of asymptotically nonexpansive mappings.

Clearly each strongly compact mapping $T: A \rightarrow E$ on a weakly compact subset $A$ of a normed space $E$ is also compact. This, together with the lemma below, shows that the class of all asymptotically semicontractive mappings on a weakly compact subset $A$ of a normed space $E$ is a subclass of the class of all weakly asymptotically semicontractive mappings on $A$.

LemMa 1.6. Let $(E,\|\cdot\|)$ be a normed space, $\varnothing \neq A \subset E$ and $S: A \times A \rightarrow$ $A$ such that

(i) $S(., x)$ is Lipschitzian with constant $L$ for all $x \in A$ and some fixed $L \geq 0$, 
(ii) $S(x,$.$) is strongly compact for each x \in A$. Then, for fixed $x \in A$ and fixed $n \in \mathbb{N}$, the mapping $y \rightarrow S(., y)^{n}(x)$ is strongly compact on $A$.

Proof. Fix $x \in A$ and define $g_{n}(y)=S(., y)^{n}(x)$ for all $y \in A$ and all $n \in \mathbb{N}$. Then, for all $n \geq 2$ and all $y, z \in A$,

$$
\begin{aligned}
\left\|g_{n}(y)-g_{n}(z)\right\| \leq & \left\|S\left(S(., y)^{n-1}(x), y\right)-S\left(S(., z)^{n-1}(x), y\right)\right\| \\
& +\left\|S\left(S(., z)^{n-1}(x), y\right)-S\left(S(., z)^{n-1}(x), z\right)\right\| \\
\leq & L\left\|S(., y)^{n-1}(x)-S(., z)^{n-1}(x)\right\| \\
& +\left\|S\left(S(., z)^{n-1}(x), y\right)-S\left(S(., z)^{n-1}(x), z\right)\right\| \\
\leq & L\left\|g_{n-1}(y)-g_{n-1}(z)\right\|+\left\|S\left(g_{n-1}(z), y\right)-S\left(g_{n-1}(z), z\right)\right\| .
\end{aligned}
$$

It follows from (5) by an easy induction that $g_{n}$ is strongly compact for each $n \in \mathbb{N}$. Indeed, $g_{1}=S(x,$.$) is strongly compact by (ii), and if g_{n}$ is strongly compact for some $n \in \mathbb{N}$, then, for each sequence $\left(z_{j}\right) \in A^{\mathbf{N}}$ weakly converging to some $z \in A$, it follows that $\lim _{j \rightarrow \infty} g_{n}\left(z_{j}\right)=g_{n}(z)$. Additionally, by (ii), $\lim _{j \rightarrow \infty} S\left(g_{n}(z), z_{j}\right)=S\left(g_{n}(z), z\right)$. Hence it follows from (5) that $\lim _{j \rightarrow \infty} g_{n+1}\left(z_{j}\right)=g_{n+1}(z)$. Consequently $g_{n+1}$ is strongly compact.

If $T$ is asymptotically semicontractive, then, in Theorem 1.5 , we are allowed to drop the assumption that $E$ possesses a weakly sequentially continuous duality mapping as we shall show in Theorem 1.8 .

LEMMA 1.7. Let $(E,\|\cdot\|)$ be a reflexive Banach space and $\varnothing \neq A \subset E$ closed bounded and convex. Suppose $S: A \times A \rightarrow A$ is such that

(i) $\left\|S\left(y_{1}, x\right)-S\left(y_{2}, x\right)\right\| \leq \lambda\left\|y_{1}-y_{2}\right\|$ for all $x, y_{1}, y_{2} \in A$ and some fixed $\lambda \in[0,1)$,

(ii) $S(x,$.$) is strongly compact for each x \in A$. Then there exists an $x \in A$ such that $S(x, x)=x$.

Proof. Let $R: A \rightarrow A$ be the mapping determined by part (a) of Lemma 1.3. It follows immediately from (ii), together with part (b) of Lemma 1.3, that $R$ is strongly compact and thus compact. Hence $R$ has a fixed point $x \in A$ by the Schauder fixed point theorem which is the desired result because $S(R x, x)=R x$.

THeOREM 1.8. Let $(E,\|\cdot\|)$ be a reflexive Banach space and $\varnothing \neq A \subset E$ closed bounded and convex. Suppose $T: A \rightarrow A$ is asymptotically semicontractive with data $\left(S,\left(k_{n}\right)\right)$ and fulfills the asymptotic regularity condition $(R)$ of Theorem 1.5. Then 
(a) $\inf \{\|x-T x\| \mid x \in A\}=0$,

(b) if (id $-T)(A)$ is closed, it follows that $F$ ix $(T) \neq \varnothing$.

Proof. It follows from Lemma 1.6 that for fixed $n \in \mathbb{N}$ and fixed $x \in$ $A$ the mapping $y \rightarrow S(., y)^{n}(x)$ is strongly compact. Thus, the proof of Theorem 1.8 is completely analogous to the proof of Theorem 1.5, except the fact that we have to use Lemma 1.7 instead of Lemma 1.4 in order to get a sequence $\left(x_{n}\right) \in A^{\mathrm{N}}$ such that $x_{n}=\left(\lambda_{n} / k_{n}\right) S\left(., x_{n}\right)^{n}\left(x_{n}\right)$ for all $n \in \mathbb{N}$.

Next, we shall extend a demiclosedness result for asymptotically nonexpansive mappings which is due to J. Gornicki [7, Lemma 4] to the wider class of asymptotically semicontractive mappings. But first we have to recall some definitions and to give two further lemmas.

Let $(E,\|\cdot\|)$ be a normed space, $\varnothing \neq A \subset E$ and $\left(x_{n}\right) \in E^{\mathrm{N}}$ bounded. For each $y \in E$ set $r\left(\left(x_{n}\right), y\right)=\lim \sup \left\|x_{n}-y\right\|$. The asymptotic radius of $\left(x_{n}\right)$ with respect to $A$ is defined by

$$
R\left(\left(x_{n}\right), A\right)=\inf _{y \in A} r\left(\left(x_{n}\right), y\right),
$$

and the asymptotic center of $\left(x_{n}\right)$ with respect to $A$ is given by

$$
A C\left(\left(x_{n}\right), A\right)=\left\{y \in A \mid r\left(\left(x_{n}\right), y\right)=R\left(\left(x_{n}\right), A\right)\right\} .
$$

LEMMA 1.9. Let $(E,\|\cdot\|)$ be a Banach space satisfying Opial's condition, $\varnothing \neq A \subset E,\left(x_{n}\right) \in E^{\mathbf{N}}$ and $x \in A$ such that $\left(x_{n}\right)-x$. Then $A C\left(\left(x_{n}\right), A\right)=$ $\{x\}$.

Proof. Just observe that it follows from [8, Lemma 3] that, for all $y \in$ $E \backslash\{x\}, \lim \sup \left\|x_{n}-x\right\|<\lim \sup \left\|x_{n}-y\right\|$.

LEMMA 1.10 [2]. Let $(E,\|\cdot\|)$ be a uniformly convex Banach space, $\varnothing \neq A \subset E$ closed and convex, $\left(x_{n}\right) \in A^{\mathrm{N}}$ bounded and $z \in A$ with $A C\left(\left(x_{n}\right), A\right)=\{z\}$. Suppose $\left(y_{n}\right) \in A^{\mathrm{N}}$ is such that

$$
\lim _{n \rightarrow \infty} r\left(\left(x_{i}\right)_{i \in \mathbb{N}}, y_{n}\right)=R\left(\left(x_{i}\right)_{i \in \mathbb{N}}, A\right) .
$$

Then $\lim \left(y_{n}\right)=z$.

THEOREM 1.11. Let $(E,\|\cdot\|)$ be a uniformly convex Banach space satisfying Opial's condition and $\varnothing \neq A \subset E$ closed and convex. Suppose $T: A \rightarrow A$ is asymptotically semicontractive with data $\left(S,\left(k_{n}\right)\right)$. Then id $-T$ is demiclosed with respect to 0 (this means that for each sequence $\left(x_{n}\right) \in A^{\mathrm{N}}$ and each point $x \in A$ such that $\left(x_{n}\right)-x$ and $\lim \left\|x_{n}-T x_{n}\right\|=0$ it follows that $T x=x)$. 
Proof. Let $\left(x_{n}\right) \in A^{\mathrm{N}}$ and $x \in A$ be such that $\left(x_{n}\right) \rightarrow x$ and $\lim \left\|x_{n}-T x_{n}\right\|=0$. For all $n, m \in \mathbb{N}$ we have

$$
\begin{aligned}
\| x_{n}- & S(., x)^{m}(x) \| \\
\leq & \left\|x_{n}-S\left(., x_{n}\right)\left(x_{n}\right)\right\|+\sum_{\nu=2}^{m}\left\|S\left(., x_{n}\right)^{\nu-1}\left(x_{n}\right)-S\left(., x_{n}\right)^{\nu}\left(x_{n}\right)\right\| \\
& +\left\|S\left(., x_{n}\right)^{m}\left(x_{n}\right)-S(., x)^{m}(x)\right\| \\
\leq & \left\|x_{n}-S\left(x_{n}, x_{n}\right)\right\|+\left\|x_{n}-S\left(x_{n}, x_{n}\right)\right\| \sum_{\nu=2}^{m} k_{\nu-1} \\
& +\left\|S\left(., x_{n}\right)^{m}\left(x_{n}\right)-S\left(., x_{n}\right)^{m}(x)\right\|+\left\|S\left(., x_{n}\right)^{m}(x)-S(., x)^{m}(x)\right\| \\
\leq & \left(1+\sum_{\nu=1}^{m-1} k_{\nu}\right)\left\|x_{n}-T x_{n}\right\|+k_{m}\left\|x_{n}-x\right\| \\
& +\left\|S\left(., x_{n}\right)^{m}(x)-S(., x)^{m}(x)\right\| .
\end{aligned}
$$

It follows from Lemma 1.6 that $y \rightarrow S(., y)^{m}(x)$ is strongly compact which in turn implies that $\lim _{n \rightarrow \infty} S\left(., x_{n}\right)^{m}(x)=S(., x)^{m}(x)$, taking into account that $\left(x_{n}\right)-x$. Thus, it follows from the inequality above that, for all $m \in \mathbb{N}$,

$$
\underset{n \rightarrow \infty}{\limsup }\left\|x_{n}-S(., x)^{m}(x)\right\| \leq k_{m} \underset{n \rightarrow \infty}{\limsup }\left\|x_{n}-x\right\|,
$$

hence

$$
r\left(\left(x_{n}\right), S(., x)^{m}(x)\right) \leq k_{m} r\left(\left(x_{n}\right), x\right) .
$$

Since $A C\left(\left(x_{n}\right), A\right)=\{x\}$ by Lemma 1.9, this leads to

$$
R\left(\left(x_{n}\right), A\right) \leq r\left(\left(x_{n}\right), S(., x)^{m}(x)\right) \leq k_{m} R\left(\left(x_{n}\right), A\right),
$$

where $\lim _{m \rightarrow \infty}\left(k_{m}\right)=1$. Thus

$$
\lim _{m \rightarrow \infty} r\left(\left(x_{n}\right), S(., x)^{m}(x)\right)=R\left(\left(x_{n}\right), A\right),
$$

from which it follows by Lemma 1.10 that $\lim _{m \rightarrow \infty} S(., x)^{m}(x)=x$. Since $S(., x)$ is continuous, this in turn implies that $S(x, x)=x$, hence $T x=x$.

Now we are in the position to prove our main result concerning the existence of fixed points of asymptotically semicontractive mappings.

THEOREM 1.12. Let $(E,\|\cdot\|)$ be a uniformly convex Banach space satisfying Opials condition and $\varnothing \neq A \subset E$ closed bounded and convex. Suppose $T: A \rightarrow A$ is asymptotically semicontractive and fulfills the asymptotic regularity condition $(R)$ of Theorem 1.5. Then there exists an $x \in A$ such that $T x=x$. 
Proof. Since every uniformly convex Banach space is reflexive (see, for example, [4, page 37]), it follows from Theorem 1.8 that there exists a sequence $\left(x_{n}\right) \in A^{\mathrm{N}}$ such that $\lim \left\|x_{n}-T x_{n}\right\|=0$. Furthermore, as a consequence of the weak compactness of $A$, there exists some subsequence $\left(x_{\varphi_{n}}\right)$ of $\left(x_{n}\right)$ which converges weakly to a point $x \in A$. Since id $-T$ is demiclosed with respect to 0 by Theorem 1.11, it follows that $x$ is a fixed point of $T$.

REMARK. With regard to [3, Theorem 1], we remark that the class of all Banach spaces possessing a weakly sequentially continuous duality mapping is properly included in the class of all Banach spaces satisfying Opial's condition (see [8]).

\section{A fixed point theorem for a special class of weakly semicontractive mappings}

In Lemma 1.4 above, as well as in [3, Theorem 2] (the analogue to Theorem 1.5 for weakly semicontractive mappings without any assumption concerning asymptotic regularity), it is assumed that $E$ possesses a weakly sequentially continuous duality mapping. This is the case, for example, if $E=l_{p}, p \in$ $(1, \infty)$, but not if $E=L_{p}[0,2 \pi], p \in(1, \infty) \backslash\{2\}$ (see [10]). Thus, it is desirable to obtain analogous results for a class of spaces which includes both the $l_{p}$ and the $L_{p}$ spaces $(p \in(1, \infty))$. Indeed, by strengthening the compactness assumption on $T$, we shall be able to establish corresponding results in Banach spaces which are uniformly smooth, a property which is shared by both $l_{p}$ and $L_{p}, p \in(1, \infty)$ (see, for example, [4, page 57]). Essential use is made of ideas from a proof of [12, Corollary 1] given in [6, pages 45-47]. The following lemma has been shown in the course of this proof.

Recall that a Banach limit LIM is a bounded linear functional on $l_{\infty}$ such that $\|\operatorname{LIM}\|=1$, and $\liminf \left(x_{n}\right) \leq \operatorname{LIM}\left(x_{n}\right) \leq \lim \sup \left(x_{n}\right)$ and $\operatorname{LIM}\left(x_{n}\right)=\operatorname{LIM}\left(x_{n+1}\right)$ for all $\left(x_{n}\right) \in l_{\infty}$.

Lemma 2.1 [6]. Let $(E,\|\cdot\|)$ be a uniformly smooth Banach space, $\varnothing \neq$ $A \subset E$ convex, $\left(x_{n}\right) \in E^{\mathbf{N}}$ bounded and LIM a Banach limit. Define $a$ mapping $f: A \rightarrow \mathbb{R}^{+}$by $f(z)=\operatorname{LIM}\left\|x_{n}-z\right\|^{2}$ for all $z \in A$, and suppose that $y \in A$ is such that $f(y)=\inf \{f(w) \mid w \in A\}$. Then

$$
\operatorname{LIM}\left(J_{E}\left(x_{n}-y\right)(z-y)\right) \leq 0
$$

for all $z \in A$. 
LemMA 2.2. Let $(E,\|\cdot\|)$ be a uniformly smooth Banach space and $\varnothing \neq$ $A \subset E$ closed bounded and convex. Suppose $S: A \times A \rightarrow A$ is such that

(i) $\left\|S\left(y_{1}, x\right)-S\left(y_{2}, x\right)\right\| \leq \lambda\left\|y_{1}-y_{2}\right\|$ for all $x, y_{1}, y_{2} \in A$ and some fixed $\lambda \in[0,1)$,

(ii) $S(x,$.$) is continuous for each x \in A$, and

(C) for each sequence $\left(x_{n}\right) \in A^{\mathrm{N}}$ there exists a subsequence $\left(x_{\varphi_{n}}\right)$ of $\left(x_{n}\right)$ such that for each $y \in A$ the strong limit $z_{y}=\lim _{n \rightarrow \infty} S\left(y, x_{\varphi_{n}}\right)$ exists in $A$.

Then there exists an $x \in A$ such that $S(x, x)=x$.

Proof. It is a consequence of (ii) and part (b) of Lemma 1.3 that the mapping $R: A \rightarrow A$ determined by part (a) of Lemma 1.3 is continuous. Thus, in view of the Schauder fixed point theorem, we are done as soon as we have shown that $R(A)$ is relatively compact (cf. the proof of Lemma 1.4).

So let $\left(x_{n}\right) \in A^{\mathrm{N}}$ and define $y_{n}=R\left(x_{n}\right)$ for all $n \in \mathbb{N}$. Take a subsequence $\left(x_{\varphi_{n}}\right)$ of $\left(x_{n}\right)$ according to (C), and denote $\lim S\left(y, x_{\varphi_{n}}\right)$ by $z_{y}$ for all $y \in$ $A$. Furthermore, fix any Banach limit LIM and define a mapping $f: A \rightarrow \mathbb{R}^{+}$ by

$$
f(z)=\operatorname{LIM}\left\|y_{\varphi_{n}}-z\right\|^{2}
$$

for all $z \in A$. Then $f$ is convex and continuous, and, taking into account that every uniformly smooth Banach space is reflexive (see, for example, $[4$, page 38]), it follows that $f$ attains its infimum over $A$ at at least one point $y \in A$ (see, for example, [16, Proposition 9.13]). Thus, by Lemma 2.1, for all $z \in A$,

$$
\operatorname{LIM}\left(J_{E}\left(y_{\varphi_{n}}-y\right)(z-y)\right) \leq 0 .
$$

Furthermore, we have already shown in the proof of Lemma 1.4 that

$$
\left\|y_{n}-y\right\|^{2} \leq\left(\frac{1}{1-\lambda}\right) J_{E}\left(y_{n}-y\right)\left(S\left(y, x_{n}\right)-y\right)
$$

for all $n \in \mathbb{N}$. Thus, for all $n \in \mathbb{N}$ and all $z \in A$,

$$
\begin{aligned}
\left\|y_{n}-y\right\|^{2} \leq & \left(\frac{1}{1-\lambda}\right) J_{E}\left(y_{n}-y\right)(z-y) \\
& +\left(\frac{1}{1-\lambda}\right) J_{E}\left(y_{n}-y\right)\left(S\left(y, x_{n}\right)-z\right),
\end{aligned}
$$

and consequently, for all $n \in \mathbb{N}$,

$$
\begin{aligned}
\left\|y_{\varphi_{n}}-y\right\|^{2} \leq & \left(\frac{1}{1-\lambda}\right) J_{E}\left(y_{\varphi_{n}}-y\right)\left(z_{y}-y\right) \\
& +\left(\frac{1}{1-\lambda}\right) \operatorname{diam}(A)\left\|S\left(y, x_{\varphi_{n}}\right)-z_{y}\right\| .
\end{aligned}
$$


Since $\lim S\left(y, x_{\varphi_{n}}\right)=z_{y}$, it follows from (6) and (7) that $\operatorname{LIM}\left\|y_{\varphi_{n}}-y\right\|^{2} \leq$ 0 , which implies that $\liminf \left\|y_{\varphi_{n}}-y\right\|=0$. Thus $\left(y_{\varphi_{n}}\right)$ possesses a subsequence $\left(y_{\varphi_{v_{n}}}\right)$ which converges strongly to $y$. Consequently $R(A)$ is relatively compact.

THEOREM 2.3. Let $(E,\|\cdot\|)$ be a uniformly smooth Banach space and $\varnothing \neq A \subset E$ closed bounded and convex. Suppose $S: A \times A \rightarrow A$ is such that

(i) $S(., x)$ is nonexpansive for each $x \in A$,

(ii) $S(x,$.$) is continuous for each x \in A$,

(iii) condition (C) of Lemma 2.2 is fulfilled.

Then, for the mapping $T: A \rightarrow A$ given by $T x=S(x, x)$,

(1) $\inf \{\|x-T x\| \mid x \in A\}=0$,

(2) if (id $-T)(A)$ is closed, it follows that $F$ ix $(T) \neq \varnothing$.

Proof. Assume, without loss of generality, that $0 \in A$ and define $\lambda_{n}=$ $1-1 / n$ for all $n \in \mathbb{N}$. Then one easily verifies that for fixed $n \in \mathbb{N}$, $S_{n}: A \times A \rightarrow A$ given by $S_{n}(x, y)=\lambda_{n} S(x, y)$ satisfies all the assumptions of Lemma 2.2. Thus, for each $n \in \mathbb{N}$, there exists an $x_{n} \in A$ such that $x_{n}=S_{n}\left(x_{n}, x_{n}\right)=\lambda_{n} T x_{n}$. Since $\left\|x_{n}-T x_{n}\right\| \leq\left|1-1 / \lambda_{n}\right| \operatorname{diam}(A)$, the result follows.

REMARK. (1) It is not difficult to see that for a mapping $S: A \times A \rightarrow A$, defined on a weakly compact subset $A$ of a normed space $E$, each of the following two conditions is sufficient to assure that supposition $(C)$ is fulfilled:

(a) $S(x,$.$) is strongly compact for each x \in A$;

(b) the mapping $\mu: A \rightarrow B(A, E):=\{g: A \rightarrow E \mid g$ is bounded $\}$ according to $\mu(x)=S(., x)$ is compact with respect to the norm topology on $A$ and the topology of pointwise convergence on $B(A, E)$.

Furthermore, if $S: A \times A \rightarrow A$ satisfies condition (C) and is such that $S(x,$.$) is continuous for each x \in A$, then $S(x,$.$) is clearly compact for$ each $x \in A$. Thus, if we neglect that in F.E. Browder's definition (see introduction) $S$ has to be defined on the whole of $E \times E$, then the class of all mappings $T: A \rightarrow A$ for which there exists a mapping $S$ corresponding to $T$ and satisfying the conditions (i), (ii) and (iii) of Theorem 2.3 is settled between the class of all semicontractive and that of all weakly semicontractive mappings.

(2) From [3, Theorem 5] we know that, in Theorem 2.3 above, there is no need to assume that $E$ is uniformly smooth, if supposition (iii) is replaced with the stronger assumption that the mapping $\mu: A \rightarrow B(A, E)$ according to $\mu(x)=S(., x)$ is compact with respect to the norm topology on $A$ and the topology of uniform convergence on $B(A, E)$. 


\section{Acknowledgement}

I wish to thank Professor Dr. J. Reinermann for his interest in this paper.

\section{References}

[1] S.C. Bose, 'Weak convergence to the fixed point of an asymptotically nonexpansive map', Proc. Amer. Math. Soc. 68 (1978), 305-308.

[2] S.C. Bose and S.K. Laskar, 'Fixed point theorems for certain class of mappings', J. Math. Phys. Sci. 19 (1985), 503-509.

[3] F.E. Browder, 'Fixed point theorems for nonlinear semicontractive mappings in Banach spaces', Arch. Rational Mech. Anal. 21 (1966), 259-269.

[4] J. Diestel, Geometry of Banach Spaces - Selected Topics, Lecture Notes in Math. 485 (Springer-Verlag, Berlin, Heidelberg, New York, 1975).

[5] K. Goebel and W.A. Kirk, 'A fixed point theorem for asymptotically nonexpansive mappings', Proc. Amer. Math. Soc. 35(1) (1972), 171-174.

[6] K. Goebel and S. Reich, Uniform Convexity, Hyperbolic Geometry, and Nonexpansive Mappings, Pure Appl. Math. Vol. 83, (Marcel Dekker, Inc., New York and Basel, 1984).

[7] J. Górnicki, 'Weak convergence theorems for asymptotically nonexpansive mappings in uniformly convex Banach spaces', Comment. Math. Univ. Carolin. 30 (2) (1989), 249-252.

[8] J.-P. Gossez and E. Lami Dozo, 'Some geometric properties related to the fixed point theory for nonexpansive mappings', Pacific J. Math. 40 (1972), 565-573.

[9] N. Hirano and W. Takahashi, 'Nonlinear ergodic theorems for nonexpansive mappings in Hilbert spaces', Kodai Math. J. 2 (1979), 11-25.

[10] Z. Opial, 'Weak convergence of the sequence of successive approximations for nonexpansive mappings', Bull. Amer. Math. Soc. 73 (1967), 591-597.

[11] G.B. Passty, 'Construction of fixed points for asymptotically nonexpansive mappings', Proc. Amer. Math. Soc. 84 (1982), 212-216.

[12] S. Reich, 'Strong convergence theorems for resolvents of accretive operators in Banach spaces', J. Math. Anal. Appl. 75 (1980), 287-292.

[13] D. Roux and S.P. Singh, 'On some fixed point theorems', Internat. J. Math. Math. Sci. 12 (1989), 61-64.

[14] J. Schu, 'Approximation of fixed points of asymptotically nonexpansive mappings', Proc. Amer. Math. Soc. 112 (1991), 143-151.

[15] P. Vijayaraju, 'Fixed point theorems for asymptotically nonexpansive mappings', Bull. Calcutta Math. Soc. 80 (1988), 133-136.

[16] E. Zeidler, Nonlinear Functional Analysis and its Applications I, Fixed-Point Theorems, (Springer-Verlag, New York, Berlin, Heidelberg, Tokyo, 1986).

Nosenberger Straße 67

D-4000 Düsseldorf 30

Federal Republic of Germany 